\title{
HOLOMORPHIC APPROXIMATION IN BANACH SPACES: A SURVEY
}

\author{
FRANCINE MEYLAN
}

Abstract. We give a survey about the Runge approximation problem for a holomorphic function defined on the unit ball of a complex Banach space.

\section{INTRODUCTION}

Given a complex Banach space $X$ of infinite dimension, we recall the following Runge approximation problems:

(1) Given $r \in(0,1), \epsilon>0$, and $f$ a holomorphic function on the open unit ball of $X$, is there an entire function $h$ satisfying $|f-h|<\epsilon$ on the open ball of radius $r$ centered at the origin? See for instance [8].

(2) Is there $r \in(0,1)$ such that for any $\epsilon>0$, and any holomorphic function $f$ on the open unit ball of $X$, there exists a entire function $h$ satisfying $|f-h|<\epsilon$ on the open ball of radius $r$ centered at the origin? See for instance [13].

We note that problem (2) is clearly independent of the choice of an equivalent norm defining the topology of $X$, while problem (1) is the analogous version of what is known to be true when $X$ is finite dimensional. The main difficulty here is due to Riesz Theorem asserting that the unit ball is not relatively compact when $\operatorname{dim} X=\infty$. In other words, we may possibly deal with holomorphic functions defined on the unit ball that are not bounded on smaller balls. The following example shows that this is indeed the case.

Example 1.1. Consider on the Banach space $l^{1}(\mathbb{N})=\left\{z: \mathbb{N} \longrightarrow \mathbb{C},\|z\|=\sum_{j=1}^{\infty}|z(j)|<\infty,\right\}$ the function given by

$$
f(z)=\sum_{j=1}^{\infty} 2^{j} z(j)^{j},
$$

and let $e_{k}$ be defined by $e_{k}(j)=\delta_{j k}$. One can check that $f$ is holomorphic on the unit ball, and since $f\left(\frac{2}{3} e_{k}\right)=2^{k}\left(\frac{2}{3}\right)^{k}, f$ is unbounded on $\overline{B\left(0, \frac{2}{3}\right)}$.

On the other hand, since unbounded holomorphic functions on the unit ball may occur, we can not replace, as in the finite dimensional case, $h$ entire holomorphic function by $h$ holomorphic polynomial in the setting of the Runge approximation problems. Indeed, holomorphic polynomials are bounded on the unit ball.

The first result in the direction of problem (1) is due to L. Lempert. He proves the following theorem for the Banach space $l^{1}(\Gamma)=\left\{z: \Gamma \longrightarrow \mathbb{C},\|z\|=\sum_{\gamma \in \Gamma}|z(\gamma)|<\infty,\right\}$ where $\Gamma$ is any set.

Analyse Complexe/Complex Analysis

The author was partially supported by Swiss NSF Grant 2100-063464.00/1. 
Theorem 1.2. 8] Let $\left(X,\left\{\varphi_{\alpha}\right\}\right)$ be a locally convex space and $M$ be a Stein manifold. Let $K \subset M$ be a compact set that is convex with respect to the set of holomorphic functions on $M$, and $V \subset M$ be an open neighborhood of $K$. Define

$$
\Omega:=\left\{(m, z) \in V \times l^{1}(\Gamma):\|z\|<R(m)\right\}, \quad \omega:=\{(m, z) \in \Omega:\|z\|<r(m)\},
$$

where $r$ and $R$ are positive continuous functions on $V$, satisfying $r<R$.

Then for every seminorm $\varphi_{\alpha}$, for every $\epsilon>0$, for every $X$-valued holomorphic map $f$ defined on $\Omega$, there exists an $X$-valued holomorphic map $g$ defined on $M \times l^{1}(\Gamma)$ such that $\varphi_{\alpha}(f-g)<\epsilon$ on $\left.\omega\right|_{K}$.

One of Lempert's motivations to get interested into the Runge approximation problems has been for instance the solvability of the $\bar{\partial}$ equation in Banach spaces. See [9], [10], [17]. In particular, he obtains the following theorem:

Theorem 1.3. [10] If $\Omega \subset l^{1}(\mathbb{N})$ is pseudoconvex and $f \in C_{0,1}(\Omega)$ is a closed locally Lipschitz continuous $(0,1)$ form, then the equation $\bar{\partial} u=f$ has a solution $u \in C^{1}(\Omega)$.

When solving the problem when $\Omega$ is the ball centered at 0 of radius $R$ in $[9$, he shows first that $\bar{\partial} u=f$ is solvable on balls centered at 0 of radius $r<R$, and then constructs a global solution on the ball centered at 0 of radius $R$ using the Runge approximation problem (1).

\section{Preliminaries}

Let $X$ be a complex Banach space. For $r>0$, we use $B(0, r)$ to denote the ball of radius $r$. Recall that a function $f: U \subset X \longrightarrow \mathbb{C}$, where $U$ is an open subset of $X$, is holomorphic if $f$ is continuous on $U$, and $f_{\mid U \cap X_{1}}$ is holomorphic, in the classical sense, as a function of several complex variables, for each finite dimensional subspace $X_{1}$ of $X$. (See [2].) We note that unlike the finite dimensional case, the continuity of $f$ is not automatic as it is shown in the following example:

Example 2.1. Let $\left\{e_{i}\right\}$ be an algebraic basis of $X$ with $\left\|e_{i}\right\|=1$, and let $f: X \longrightarrow \mathbb{C}$ be the linear form satisfying $f\left(e_{i}\right)=i$. Since $f$ is not bounded, $f$ is not holomorphic.

We now recall the definition of a holomorphic polynomial.

Definition 2.2. Let $\Delta_{n}: X \longrightarrow X^{n}$ be the mapping defined by $\Delta_{n}(x)=(x, \ldots, x)$. A n-homogeneous holomorphic polynomial $P: X \longrightarrow \mathbb{C}$ is the composition of $\Delta_{n}$ with any continuous $n$-linear map $L$, that is, $P=L \circ \Delta_{n}$. A holomorphic polynomial is a finite sum of homogeneous holomorphic polynomials.

Definition 2.3. Let $H$ be a holomorphic function on $B(0, r), r>0$. We say that $H$ is $n$-homogeneous if $H(\lambda x)=\lambda^{n} H(x)$ for $\lambda \in \mathbb{C},|\lambda| \leq 1$.

For the convenience of the reader, we give the proof of the next propositions.

Proposition 2.4. Let $f_{n}: B(0, r) \longrightarrow \mathbb{C}$ be holomorphic functions such that $\lim _{n \rightarrow \infty} f_{n}=f$, uniformly on compact subsets of $B(0, r)$. Then $f$ is holomorphic on $B(0, r)$.

Proof. Since $f$ is clearly holomorphic when restricted to finite dimensional subspaces of $X$, we only have to show the continuity of $f$. Let $\left\{z_{n}\right\}$ be a sequence in $X$ with $\lim _{n \rightarrow \infty} z_{n}=z \in X$. By assumption, given $\epsilon>0$, there exists on the compact subset $\left\{\left\{z_{n}\right\}, z\right\}, M$ depending only on $\epsilon$ such that

$$
\left|f(z)-f_{M}(z)\right|+\left|f_{M}\left(z_{n}\right)-f\left(z_{n}\right)\right|<\frac{2}{3} \epsilon .
$$


Hence, there exists $N>0$ such that for $n \geq N$

$$
\left|f(z)-f\left(z_{n}\right)\right| \leq\left|f(z)-f_{M}(z)\right|+\left|f_{M}(z)-f_{M}\left(z_{n}\right)\right|+\left|f_{M}\left(z_{n}\right)-f\left(z_{n}\right)\right|<\epsilon .
$$

Proposition 2.5. Let $H: B(0, r) \longrightarrow \mathbb{C}$ be a n-homogeneous holomorphic function. Then $H$ is an entire function, that is a holomorphic function defined on $X$.

Proof. For $z \in X$, we may write $z=\lambda_{1} z_{1}, z_{1} \in B(0, r)$. We define $\tilde{H}(z):=\lambda_{1}{ }^{n} H\left(z_{1}\right)$ and claim that it is a well defined entire function. Indeed if $z$ has another representation $z=\lambda_{2} z_{2}, z_{2} \in B(0, r)$, then, without loss of generality, we will have $z_{1}=\frac{\lambda_{2}}{\lambda_{1}} z_{2}, \quad\left|\frac{\lambda_{2}}{\lambda_{1}}\right| \leq 1$, which implies $H\left(z_{1}\right)=H\left(\frac{\lambda_{2}}{\lambda_{1}} z_{2}\right)$.

Proposition 2.6. Let $H: X \longrightarrow \mathbb{C}$ be a n-homogeneous holomorphic function. Then $H$ is a $n$-homogeneous holomorphic polynomial. More precisely, there is a unique symetric continuous $n$-linear map L such that $H=L \circ \Delta_{n}$.

Proof. If $L$ exists, it has to satisfy

$$
\begin{gathered}
H\left(z_{1} x_{1}+\cdots+z_{n} x_{n}\right)=L\left(\sum z_{j} x_{j}, \ldots, \sum z_{j} x_{j}\right)= \\
z_{1}^{n} L\left(x_{1}, \ldots, x_{1}\right)+\cdots+n ! z_{1} z_{2} \ldots z_{n} L\left(x_{1}, \ldots, x_{n}\right)+\cdots+z_{n}{ }^{n} L\left(x_{n}, \ldots, x_{n}\right) .
\end{gathered}
$$

Hence, we have the integral formula

$$
L\left(x_{1}, \ldots, x_{n}\right)=\frac{1}{n !}(2 \pi i)^{-n} \int_{\left|z_{1}\right|=\epsilon_{1}} \ldots \int_{\left|z_{n}\right|=\epsilon_{n}} \frac{H\left(z_{1} x_{1}+\cdots+z_{n} x_{n}\right)}{z_{1}^{2} \ldots z_{n}^{2}} d z_{1} \ldots d z_{n} .
$$

Using (2.4), we obtain the uniqueness. For the existence, we use (2.4) as a definition and show that $L$ is a (symetric) $n$-linear map using a linear change of variables, the continuity of $L$ beeing obtained by using $\epsilon_{j}$ small enough.

\section{BOUNDED HOLOMORPHIC FUNCTIONS}

We start this section by recalling the following definition:

Definition 3.1. Let $f: B(0,1) \longrightarrow \mathbb{C}$ be a holomorphic function. We define $f_{n}(z)$ as

$$
f_{n}(z):=\int_{0}^{2 \pi} f\left(e^{2 \pi i t} z\right) e^{-2 \pi i n t} d t
$$

Lemma 3.2. Let $f: B(0,1) \longrightarrow \mathbb{C}$ be a holomorphic function. Then $f_{n}$ defined by (3.1) is a n-homogeneous holomorphic polynomial.

Proof. We first notice that $f_{n}$ is a holomorphic fonction on the unit ball that is $n$-homogeneous along any finite dimensional subspace. We conclude then, using Proposition 2.6, that $f_{n}$ is a $n$-homogeneous holomorphic polynomial.

Lemma 3.3. Let $f: B(0,1) \longrightarrow \mathbb{C}$ be a holomorphic function. Then

$$
f(z)=\sum_{n=1}^{\infty} f_{n}(z)
$$

Proof. The proof is achieved by restricting to finite dimensional subspaces of $X$. 
The following theorem shows that uniform approximation by holomorphic polynomials is possible for bounded holomorphic functions. For the convenience of the reader, we give the proof.

Theorem 3.4. Let $f: B(0,1) \longrightarrow \mathbb{C}$ be a holomorphic function that is bounded on smaller balls $B(0, r), 0<r<1$. Then given $\epsilon>0$, there exists a holomorphic polynomial $h$ satisfying $|f-h|<\epsilon$ on $B(0, r)$.

Proof. We claim that, for $0<\sigma<1$, we have

$$
\varlimsup_{n \rightarrow \infty} \sigma\left(\left|f_{n}\right|_{B(0,1)}\right)^{\frac{1}{n}}<1 .
$$

Indeed, since $f$ is bounded on $B(0,1)$, we have $M \geq\left|f_{n}(\sigma z)\right|=\sigma^{n}\left|f_{n}(z)\right|$. Hence $\sigma\left|f_{n}(z)\right|^{\frac{1}{n}}<M^{\frac{1}{n}}$, which implies $\varlimsup_{n \rightarrow \infty} \sigma\left(\left|f_{n}\right|_{B(0,1)}\right)^{\frac{1}{n}} \leq 1$. Choosing $\tilde{\sigma}<1$ satisfying $\tilde{\sigma}=\lambda \sigma, \lambda>1$, we obtain (3.3). We then consider, for $r<1$, the ball $B(0, r)=r B(0,1)$. Using (3.3), we obtain that there exists $\mu<1$ such that $r^{n}\left|f_{n}\right|_{B(0,1)}<\lambda^{n}$, for $n$ large enough. Using Lemma 3.3, we then conclude that $f(z)=\sum_{n=1}^{\infty} f_{n}(z)$ uniformly on $B(0, r)$. The proof of the proposition is achieved using Lemma 3.2

\section{A Counterexample}

In this section, we discuss the counterexample given by Lempert in [14]. We start with the following definition:

Definition 4.1. A set $S$ is called a bounding set if $\|f\|_{S}<\infty$ for every entire function.

In [1], Dineen shows that $l^{\infty}$ admits non compact closed bounding subsets. More precisely, he shows that any entire function on $l^{\infty}$ is bounded on the set $S=\left\{e_{n}\right\}$, where $e_{n}(j)=\delta_{j n}$.

Lempert considers a sequence of norms on $l^{\infty}$, all equivalent to the sup norm. More precisely, he defines

$$
\|z\|_{k}=\frac{2}{k} \sup _{j_{1}<j_{2}<\cdots<j_{k}}\left|z\left(j_{1}\right)\right|+\left|z\left(j_{2}\right)\right|+\cdots+\left|z\left(j_{k}\right)\right|, \quad z: \mathbb{N}: \longrightarrow \mathbb{C} \in l^{\infty} .
$$

Then he considers on $\left(l^{\infty},\|z\|_{k}\right)$, the following holomorphic function on the unit ball

$$
f(z)=\sum_{j=1}^{\infty} j z(j)^{j},
$$

that is unbounded on the set $S=\left\{e_{n}\right\}$. This shows, using Dineen's result, that the answer to Problem (1) and Problem (2) is negative on $\left(l^{\infty},\|z\|_{k}\right)$. We refer the interested reader to [14 for the details.

\section{The General CASE}

In the light of the counterexample given by Lempert, we may ask the following question:

- Do Runge approximations (1) and (2) hold in any separable Banach space?

Of particular interest is the space $C[0,1]$ since every separable Banach space is isometric to a subspace of $C[0,1]$. The Runge approximations are still open for this space as well as for the Banach space $L^{1}(0,1)$. As said in the introduction, the obstruction to Runge approximations is Riesz Theorem. Therefore, one needs to look for "good" compact subsets that "replace" the unit ball. The following lemma gives some understanding of what kind of sufficient conditions is needed to obtain a positive answer to Problem (1) and Problem (2). 
Lemma 5.1. 3] Let $T_{n}$ be a uniformly bounded sequence of linear operators in $X$. If $\lim _{n} T_{n} x=x$ for every $x \in X$, then this limit exists uniformly on any compact set. Conversely, if $\lim _{n} T_{n} x=x$ uniformly for $x$ in a bounded set $K$, and if, in addition, $T_{n} K$ is relatively compact for each $n$, then $K$ relatively is compact.

We recall the following definitions.

Definition 5.2. A series $\sum_{n=1}^{\infty} x_{n}$ is said to converge unconditionally if $\sum_{n=1}^{\infty} x_{\pi(n)}$ converges for every permutation $\pi$ of the integers.

Definition 5.3. Let $X$ be a complex Banach space. A sequence $\left\{X_{n}\right\}$ of closed subspaces of $X$ is called a Schauder decomposition of $X$ if every $x \in X$ has a unique representation of the form $x=\sum_{n=1}^{\infty} x_{n}$, with $x_{n} \in X$. The Schauder decomposition is unconditional if for every $x \in X$, the series $\sum_{n=1}^{\infty} x_{n}$ which represents $x$ converges unconditionally.

Remark 5.4. [18] A decomposition $\left\{X_{n}\right\}$ of a Banach space $X$ is a Schauder decomposition of $X$ if and only if the projections $\left\{P_{j}\right\}$ defined by

$$
P_{j} \sum_{n=1}^{\infty} x_{n}=\sum_{n=1}^{j} x_{n}
$$

are continuous. Moreover $\left\{P_{j}\right\}$ is a uniformly bounded sequence of linear operators in $X$.

Example 5.5. $l^{p}(\mathbb{N})=\left\{z: \mathbb{N} \longrightarrow \mathbb{C},\|z\|=\left(\sum_{j=1}^{\infty}|z(j)|^{p}\right)^{\frac{1}{p}}<\infty\right\}$ admits an unconditional Schauder basis, i.e. $\operatorname{dim} X_{n}=1$.

Example 5.6. Let $X$ be the space of compact operators on $l^{2}(\mathbb{N})$ which have a triangular representing matrix with respect to $\left\{e_{n}\right\}$, where $e_{n}(j)=\delta_{j n}$. Let $X_{n}$ be the subspace of $X$ defined as

$$
X_{n}=\left\{T \in X \mid T e_{j}=0, j \neq n\right\} .
$$

Then $\left\{X_{n}\right\}$ is an unconditional Schauder decomposition of $X$ with $\operatorname{dim} X_{n}<\infty$. In this case, we say that $X$ has a UFDD (unconditional finite dimensional decomposition). Moreover, by a result of Gordon and Lewis, $X$ does not have an unconditional Schauder basis. See [4], [16].

Example 5.7. It is well known that the space of compact operators on $l^{2}(\mathbb{N})$ has an unconditional Schauder decomposition into UFDD, but has no UFDD itself. See 7].

Assume now that $X$ admits an unconditional Schauder decomposition $\left\{X_{n}\right\}_{n=1}^{\infty}$. Let $x=\sum_{n=1}^{\infty} x_{n}$, $x_{n} \in X_{n}$, be the unique representation of $x$. It is known that for every sequence of complex numbers $\theta=\left\{\theta_{n}\right\},\left|\theta_{n}\right| \leq 1, n \in \mathbb{N}$, the operator $M_{\theta}$ defined by

$$
M_{\theta} \sum_{n=1}^{\infty} x_{n}=\sum_{n=1}^{\infty} \theta_{n} x_{n}
$$

is a bounded linear operator. The (finite) constant $\sup _{\theta}\left\|M_{\theta}\right\|$ is called the unconditional constant of the decomposition.(See [16] and [18] for details). It is clear that one can always define on $X$ an equivalent norm \|\|$_{1}$ so that the unconditional constant becomes 1. (Take $\left.\|x\|_{1}=\sup _{\theta}\left\|M_{\theta} x\right\|\right)$. In other words, we have

$$
\left\|\sum_{n=1}^{\infty} \theta_{n} x_{n}\right\|_{1} \leq\left\|\sum_{n=1}^{\infty} x_{n}\right\|_{1}, \quad\left|\theta_{n}\right| \leq 1, n \in \mathbb{N} .
$$


Using Lemma 5.1 and (5.2), one proves

Proposition 5.8. Let $X$ be a complex Banach space admitting an unconditional Schauder decomposition $\left\{X_{n}\right\}$ with unconditional constant one. Then the following holds.

(1) $M_{\theta}(B(0, R))$ is relatively compact in $B(0, R)$ for any sequence of complex numbers $\theta=\left\{\theta_{n}\right\},\left|\theta_{n}\right|<$ 1 , which converges to 0 if and only if $\operatorname{dim} X_{n}<\infty$, for any $n$.

(2) For any compact $K \subset B(0, R)$, there exists a sequence of complex numbers $\theta=\left\{\theta_{n}\right\},\left|\theta_{n}\right|<1$ which converges to 0 , and a compact $L \subset B(0, R)$ so that $M_{\theta} L=K$.

Proof. We note that $P_{j}\left(M_{\theta}(B(0, R))\right)$ is relatively compact if and only if $\operatorname{dim} X_{n}<\infty$, for any $n$. The rest of the proof is similar to the one of Proposition 1.2 in [11].

Remark 5.9. The sets $M_{\theta}(B(0, R))$ in Proposition 5.8 will play the role of "good"' relatively compact sets that replace the unit ball in the case of a space admitting a UFDD.

\section{The case of Banach spaces admitting a UFDD}

In the light of the proof of Theorem 3.4, Remark 5.9 leads to the following definitions that appear in [8, [11, [5] and [13].

Let $X$ be a complex Banach space admitting an unconditional Schauder decomposition $\left\{X_{n}\right\}$ with unconditional constant one. Let $z \in X$ be given by its unique representation $z=\sum_{n=1}^{\infty} z_{n}$, with $z_{n} \in X_{n}$ for every $n$. For $m \in \mathbb{N}$, one defines

$$
T(m):=\left\{e^{2 \pi i t}, t=\left\{t_{n}\right\}, t_{n} \in \mathbb{R}, t_{n}=0 \text { for } n>[\sqrt{m}]\right\}
$$

and

$$
K(m):=\left\{k=\left\{k_{n}\right\}, k_{n} \in \mathbb{N} \cup\{0\}, k_{n}=0 \text { for } n>[\sqrt{m}], \sum_{n=1}^{[\sqrt{m}]} k_{n} \leq m\right\}
$$

Definition 6.1. Let $f$ be a holomorphic function on $B(0, R)$. For $k \in K(m)$ and $e^{2 \pi i s} \in T(m)$, we define $f^{k}(z)$ as

$$
f^{k}(z):=\int_{T(m)} f\left(M_{e^{2 \pi i s}} z\right) e^{-2 \pi i k . s} d s
$$

where $d s$ is the normalized Haar measure on $T(m)$.

Note that $f^{k}$ is homogeneous of degree $k_{n}$ in $z_{n}$.

Definition 6.2. The formal series associated to $f$ given by

$$
\sum_{m=0}^{\infty} \sum_{k \in K(m)}\left(f_{m}\right)^{k}(z)
$$

is called the Josefson series.

We have the following proposition:

Proposition 6.3. [13] Let $X$ be a complex Banach space admitting an unconditional Schauder decomposition $\left\{X_{n}\right\}$ with unconditional constant one. Then the Josefson series converges to $f$, uniformly on compact sets of $B(0, R)$ if $\operatorname{dim} X_{n}<\infty$ for any $n$. 
Remark 6.4. In Proposition 6.3, the proof of the uniform convergence on compact sets relies on the fact that the sets $M_{\theta}(B(0, R))$ are compact. It would be interesting to know if it holds without the assumption on the dimension of each $X_{n}$.

Making use of the Josefson series, one can show that problem (2) holds in spaces admitting an unconditional Schauder decomposition $\left\{X_{n}\right\}$ with $\operatorname{dim} X_{n}<\infty$ for any n. More precisely, we have

Theorem 6.5. 13] Let $X$ be a complex Banach space admitting an unconditional Schauder decomposition $\left\{X_{n}\right\}$ with $\operatorname{dim} X_{n}<\infty$ for any $n$. Then there exists an equivalent norm \|\| on $X$ for which the Runge approximation problem (1) holds.

We may ask the following question

- Does the Runge approximation problem (2) hold in complex Banach spaces admitting an unconditional Schauder decomposition into UFDD?

Note that the "model" case of such a space is given by example (5.7).

Remark 6.6. It is known that the spaces $C[0,1]$ and $L^{1}[0,1]$ do not admit any unconditional Schauder decomposition into UFDD. Indeed, Lindenstrauss and Pelczynski in [15] showed that if $\left(X_{n}\right)$ is an unconditional Schauder decomposition of $C[0,1]$, then at least one $X_{n} \approx C[0,1]$, which is impossible [18. N.J. Kalton in [6] showed that the same holds for $L^{1}[0,1]$.

\section{REFERENCES}

[1] Dineen, S. - Bounding subsets of a Banach space. Math. Ann. 192 (1971), 61-70.

[2] Dineen, S. - Complex Analysis on Infinite Dimensional Spaces. Springer, Berlin. (1999).

[3] Dunford, N.; Schwartz, T. - Linear Operators I. John Wiley and Sons, New York. (1988).

[4] Gordon, Y., Lewis, D.R. - Absolutely summing operators and local unconditional structures. Acta Math. 133 (1974), 27-48.

[5] Josefson, B. - Approximation of holomorphic functions in certain Banach spaces. International Journal of Mathematics. 15 (2004), no.5, 467-471.

[6] Kalton, N.J. - The endomorphisms of $L_{p}$. Indiana University Mathematics Journal. 27 (1978), no.3, $353-381$.

[7] Kwapien, S., Pelczynski, A. - The main triangle projection in matrix spaces and its applications. Studia mathematica. 34 (1970), 43-68.

[8] Lempert, L. - Approximation de fonctions holomorphes d'un nombre infini de variables. Ann. Inst. Fourier, Grenoble. 49 (1999), no.4, 1293-1304.

[9] Lempert, L. - The Dolbeaut complex in infinite dimensions, II. Journal of the American Mathematical Society. 12 (1999), 775-793. Ann. Inst. Fourier, Grenoble. 49 (1999), no.4, 1293-1304.

[10] Lempert, L. - The Dolbeaut complex in infinite dimensions, III. Invent. Math. 142 (2000), 579-603.

[11] Lempert, L. - Approximation of holomorphic functions of infinitely many variables. Ann. Inst. Fourier, Grenoble. 50 (2000), no. 2, 423-442.

[12] Lempert, L. - Seminar given at Purdue University. (2004).

[13] Meylan, F. - Approximation of holomorphic functions in Banach spaces admitting a Schauder decomposition. Ann. Scuola Norm. Sup. Pisa, V (2006), 13-19.

[14] Lempert, L. - A note on holomorphic approximation in Banach spaces. Periodica mathematica hungarica. 56 (2008), no. 2, 241-245.

[15] Lindenstrauss, J.; Pelczynski, A. - Contributions to the theory of classical Banach Spaces. J. Functional Analysis 8 (1971), 225-249.

[16] Lindenstrauss, J.; Tzafriri, L. - Classical Banach Spaces I, Sequence Spaces. Springer-Verlag, Berlin Heidelberg New York. 92 (1977).

[17] Patyi, I. - On the $\bar{\partial}$-equation in a Banach space. Bull. Soc. math. France. 128 (2000), 391-406.

[18] Singer, I. - Bases in Banach spaces I-II. Springer, Berlin. (1981). 
F. Meylan : Institut de Mathématiques, Université de Fribourg, 1700 Perolles, Fribourg, Switzerland

E-mail address: francine.meylan@unifr.ch 